\title{
A Parameter Estimation and Identifiability Analysis Methodology Applied to a Street Canyon Air Pollution Model
}

Thor-Bjørn Ottosen ${ }^{\mathrm{a}, \mathrm{c}}$, Matthias Ketzel ${ }^{\mathrm{b}}$, Henrik Skov ${ }^{\mathrm{b}, \mathrm{c}}$, Ole Hertel ${ }^{\mathrm{b}}$, Jørgen Brandt $^{\mathrm{b}}$, Konstantinos E. Kakosimos ${ }^{\mathrm{a}, *}$

${ }^{a}$ Department of Chemical Engineering, Texas A\&M University at Qatar, Doha, Qatar

${ }^{b}$ Department of Environmental Science, Aarhus University, Roskilde, Denmark

${ }^{c}$ Department of Chemical Engineering, Biotechnology and Environmental Technology, University of Southern Denmark, Odense, Denmark

\begin{abstract}
Mathematical models are increasingly used in environmental science thus increasing the importance of uncertainty and sensitivity analyses. In the present study, an iterative parameter estimation and identifiability analysis methodology is applied to an atmospheric model - the Operational Street Pollution Model $\left(\mathrm{OSPM}^{\circledR}\right)$. To assess the predictive validity of the model, the data is split into an estimation and a prediction data set using two data splitting approaches and data preparation techniques (clustering and outlier detection) are analysed. The sensitivity analysis, being part of the identifiability analysis, showed that some model parameters were significantly more sensitive than others. The application of the determined optimal parameter values was shown to succesfully equilibrate the model biases among the individual streets and species. It was as well shown that the frequentist approach applied for the uncertainty calculations underestimated the parameter uncertainties. The model parameter uncertainty was qualitatively assessed to be significant, and reduction strategies were identified. Keywords: Uncertainty, Sensitivity, OSPM, Data splitting, Exploratory data analysis, Matlab
\end{abstract}

${ }^{*}$ Corresponding author. tel.: +974 44230678

E-mail addresses: thor-bjorn.ottosen@qatar.tamu.edu (T.-B. Ottosen), mke@envs.au.dk (M Ketzel), hsk@envs.au.dk (H.Skov), oh@envs.au.dk (O. Hertel), jbr@envs.au.dk (J. Brandt), k.kakosimos@qatar.tamu.edu (K. E. Kakosimos). 


\section{Introduction}

A few decades ago, the use of mathematical models was mainly limited to the use internally in the scientific community, meaning that the model users to a larger extend had an explicit or implicit understanding of the model uncertainty and sensitivity ${ }^{1}$. Today, mathematical models are often routinely used by engineers, consultants, and planners as well as scientists for environmental regulation and to assess consequences of abatement strategies. This development supports the need for explicit uncertainty and sensitivity analyses to facilitate the communication among model stakeholders.

Within air pollution modelling there has been a growing number of publications on uncertainty and sensitivity analyses in recent years (for a review see Hanna (2007)). Walker et al. (2003) defined six uncertainty categories, based on the location of the uncertainty, of which some have been studied within air pollution modelling. Model technical uncertainty (e.g. Franke et al. (2007)) and model input uncertainty (Bei et al., 2012; Hanna et al., 2007; Manomaiphiboon and Russell, 2004) have been studied previously, however, model parameter uncertainty has received comparatively little attention (e.g. Marsik and Johnson (2010))

Vardoulakis et al. (2002) studied the local sensitivity of the Operational Street Pollution Model (OSPM $\left.{ }^{\circledR}\right)$ to marginal changes in ten model parameters for an artificial dataset (parallel and perpendicular wind directions and a constant wind speed). Silver et al. (2013) analysed the applicability of a dynamic parameter estimation (Parameter estimates change along with changes in data) scheme to OSPM for planning and forecasting. Secondarily, Silver et al. (2013) showed in a preliminary application of static parameter estimation (One set of parameters are estimated for all data points) that such an approach can be informative. Silver et al. (2013) used between one and four years of data for five streets. The parameter estimation scheme in Silver et al. (2013) was applied to one model parameter and five multiplicative adjustment factors. This study can thus be classified as being somewhere between analysis of model input and

\footnotetext{
${ }^{1}$ The analysis of change in model output of marginal changes in one model parameter at a time. This is opposed to global sensitivity analysis which is the analysis of change in model output of large changes of several model parameters at a time.
} 
model parameter uncertainty. The choice of model parameters and multiplicative adjustment factors was based on the developers experience with the model. A natural question is thus whether a more systematic approach would yield better results?

Brun et al. $(2002,2001)$ developed a systematic parameter estimation and identifiability analysis methodology, which has been applied among others in lake modelling (Omlin et al., 2001), river modelling (Anh et al., 2006; Meier and Reichert, 2005), modelling of waste-water treatment plants (Sin and Vanrolleghem, 2007b), forest modelling (De Pauw et al., 2008), surface hydrology modelling (Freni et al., 2009; Muñoz et al., 2014), and material science (MartinezLopez et al., 2015) but has not been applied within atmospheric science before.

To analyse the applicability of, and to explore the potential advantages of, applying this methodology to a model within atmospheric science, the methodology of Brun et al. $(2002,2001)$ was applied to the Operational Street Pollution Model $\left(\mathrm{OSPM}^{\circledR}\right)$. The application utilizes more years of data and more parameters compared to the analysis performed in Silver et al. (2013).

This paper explains the appropriate data preparation techniques, reports the results of the application of, and explores the advantages of this methodology through exploratory data analysis of the results.

The working principles behind OSPM are described in Section 2. The model input, the measurements, and the methodologies for data preparation, parameter estimation, and identifiability analysis are likewise explained in Section 2. The results and discussion of the various sub-analyses performed in the present study are presented in Section 3. The conclusions are subsequently presented in Section 4.

\section{Model description and Methods}

The applied methodology, as illustrated on Fig. 1, consists of running parameter estimation and identifiability analysis in an iterative series. This is done until convergence between the obtained parameters and the identifiability of the parameters is achieved. Following the steps outlined in Fig. 1, the model definition has been done in Berkowicz et al. (1997); Hertel and Berkowicz (1989a,b,c) as briefly described in Section 2.1. The experimental layout is defined by the 


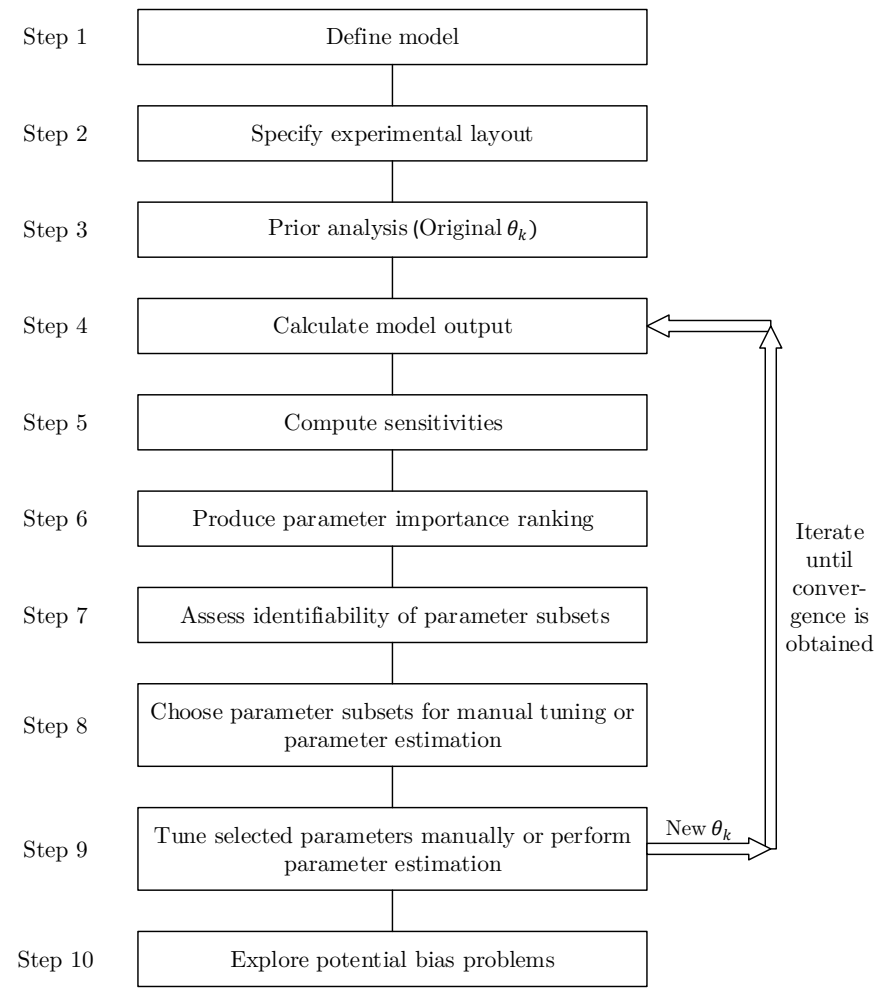

Figure 1: Schematic representation of the parameter estimation and identifiability analysis methodology applied in the present study (figure based on Brun et al. (2002)). $\theta_{k}$ is the model parameter vector. 
data available through the Danish national air quality monitoring programme and as part of the prior analysis, data preparation has been performed.

\subsection{Model description}

OSPM is a model for vehicle induced urban street pollution. The model is designed to take differences in atmospheric conditions and types of street into account. The main characteristics of OSPM are:

- The applied version of OSPM consists of emissions calculated with COPERT IV (EMEP/EEA, 2009) and a dispersion model running in series. To limit the scope of the present study the focus is on the parameters related to the dispersion model.

- OSPM models the resulting hourly averaged pollution concentrations, of a specific species, at the side of the street. This is calculated as a sum of a direct contribution $\left(C_{\mathrm{dir}}\right)$ and a recirculating contribution $\left(C_{\mathrm{rec}}\right)$ plus

a background concentration. The direct contribution is modelled using a simplified Gaussian plume model with a top hat distribution applied to the emission plume. The recirculating contribution is modelled using a trapezium shaped box model (Berkowicz, 2000; Berkowicz et al., 1997; Hertel and Berkowicz, 1989b).

- The wind direction, especially for low wind speeds, cannot be assumed constant over a full hour. To account for this, a numerical wind direction averaging procedure is implemented in the model (Hertel and Berkowicz, 1989c).

- The model also contains an algebraic expression for traffic produced turbulence. The expression depends on the number of cars in the street, their respective driving speeds, and the traffic composition (Hertel and Berkowicz, 1989c).

- Most traffic pollutants are assumed to be inert on the time scale of the residence time in a street canyon. However, the conversion of $\mathrm{NO}$ to $\mathrm{NO}_{2}$ in the presence of ozone happens faster. It is therefore included in the model in the form of an algebraic chemical conversion scheme (Hertel and Berkowicz, 1989a; Palmgren et al., 1996). The majority of the parameters 
of the chemical conversion module are left out of the subsequent parameter estimation to limit the scope of the study.

A total of 16 model parameters have been identified in the model. These are briefly summarized in Table 1. A more detailed description of the model can be found at www.au.dk/ospm or in Berkowicz et al. (1997); Ottosen et al. (2015).

\subsection{Model Inputs}

The concentration and meteorology input data come from the Danish national air quality monitoring programme (Ellermann et al., 2013). In this programme hourly air quality measurements have been performed since 1994. Measurements are performed in two streets in Copenhagen (Jagtvej and H. C. Andersens Boulevard (hereafter referred to as HCAB)) and in one street in respectively Aarhus, Aalborg, and Odense. A map of the streets can be found in Silver et al. (2013) and the characteristics of the five streets are summed up in Table 2. An analysis of the representativity of the streets for other streets in the cities can be found in the supplementary material. Details of the number of input data as a function of wind speed and atmospheric stability can likewise be found in the supplementary material.

It was important to include several pollutants in the analysis in order to prevent that optimal model performance for one species leads to poorer model performance for other species. Since $\mathrm{NO}_{2}$ is both directly emitted and converted from NO, in the presence of ozone, it will have a different set of biases compared to $\mathrm{NO}_{x} . \mathrm{NO}_{x}$ is treated as a separate species, since the group of $\mathrm{NO}$ and $\mathrm{NO}_{2}$ can thus be assumed to be inert. For these two compounds $\left(\mathrm{NO}_{x}\right.$ and $\mathrm{NO}_{2}$ ) it has been shown that the ratio is not the same in the model and the measurements (Ketzel et al., 2012). Both species were thus included in the analysis to counterbalance the biases of the other species.

$\mathrm{NO}_{x}$ and $\mathrm{NO}_{2}$ are measured continuously in the streets and at urban background stations in the four cities. $\mathrm{NO}_{2}$ and $\mathrm{NO}_{x}$ are measured by chemiluminescence on Aerodyne API instruments. The detection limits are 200 pptv and 300 pptv respectively for $\mathrm{NO}_{x}$ and $\mathrm{NO}_{2}$. The measurement uncertainty for $\mathrm{NO}_{x}$ 
Table 1: Table of model parameters in OSPM version 5.2.15 (June 2014).

\begin{tabular}{|c|c|c|}
\hline Parameter: & Initial value: & Description: \\
\hline$\alpha$ & 0.1 & $\begin{array}{l}\text { Slope of emission dispersion plume. Pro- } \\
\text { portion between roof level wind speed } \\
\text { and roof level vertical turbulence. Ele- } \\
\text { ment of denominator in the calculation } \\
\text { of chemical residence time. }\end{array}$ \\
\hline$c$ & 2.0 & $\begin{array}{l}\text { Length of recirculation zone divided by } \\
\text { the upwind building height for wind } \\
\text { speeds higher than } g \text {. }\end{array}$ \\
\hline$L_{t}$ & 0.5 & $\begin{array}{l}\text { Upper length of the recirculation trapez- } \\
\text { ium divided by the length of the baseline. }\end{array}$ \\
\hline$d$ & 0.5 & $\begin{array}{l}\text { Angle of integration in radians for wind } \\
\text { speeds higher than } i \text {. }\end{array}$ \\
\hline$f_{\text {roof }}$ & 0.4 & $\begin{array}{l}\text { Scale factor to reduce the wind speed } \\
\text { from a meteorological mast to roof level. }\end{array}$ \\
\hline$h_{0}$ & 2.0 & $\begin{array}{l}\text { Initial dispersion height in the wake of } \\
\text { a car. }\end{array}$ \\
\hline$z_{0}$ & 0.6 & $\begin{array}{l}\text { Aerodynamic roughness height used to } \\
\text { relate roof level wind to street level wind } \\
\text { in a logarithmic profile. }\end{array}$ \\
\hline$g$ & $\frac{\mathrm{m}}{\mathrm{s}}$ & $\begin{array}{l}\text { Wind speed where the recirculation zone } \\
\text { reaches its full extent. }\end{array}$ \\
\hline$i$ & $\frac{\mathrm{m}}{\mathrm{s}}$ & $\begin{array}{l}\text { Upper limit for increased wind direction } \\
\text { averaging. }\end{array}$ \\
\hline$j$ & 180.0 & $\begin{array}{l}\text { Upper limit of interval for which the } \\
\text { general building height is taken as the } \\
\text { average. }\end{array}$ \\
\hline$H_{\min }$ & 5.0 & Minimum general building height. \\
\hline$S_{p}$ & $\mathrm{~m}^{2}$ & $\begin{array}{l}\text { Aerodynamic frontal area of light duty } \\
\text { vehicles. }\end{array}$ \\
\hline$S_{t}$ & 16.0 & $\begin{array}{l}\text { Aerodynamic frontal area of heavy duty } \\
\text { vehicles. }\end{array}$ \\
\hline$b$ & 0.3 & $\begin{array}{l}\text { Scale factor for traffic produced turbu- } \\
\text { lence. }\end{array}$ \\
\hline$k$ & 0.4 & $\begin{array}{l}\text { Scale factor to reduce the impact of traf- } \\
\text { fic produced turbulence at the top of } \\
\text { the street canyon. Element in the de- } \\
\text { nominator in the calculation of chemical } \\
\text { residence time. }\end{array}$ \\
\hline$\gamma$ & 0.2 & $\begin{array}{l}\text { Scale factor for ground level wind speed } \\
\text { reduction from parallel to perpendicular } \\
\text { wind directions. }\end{array}$ \\
\hline
\end{tabular}




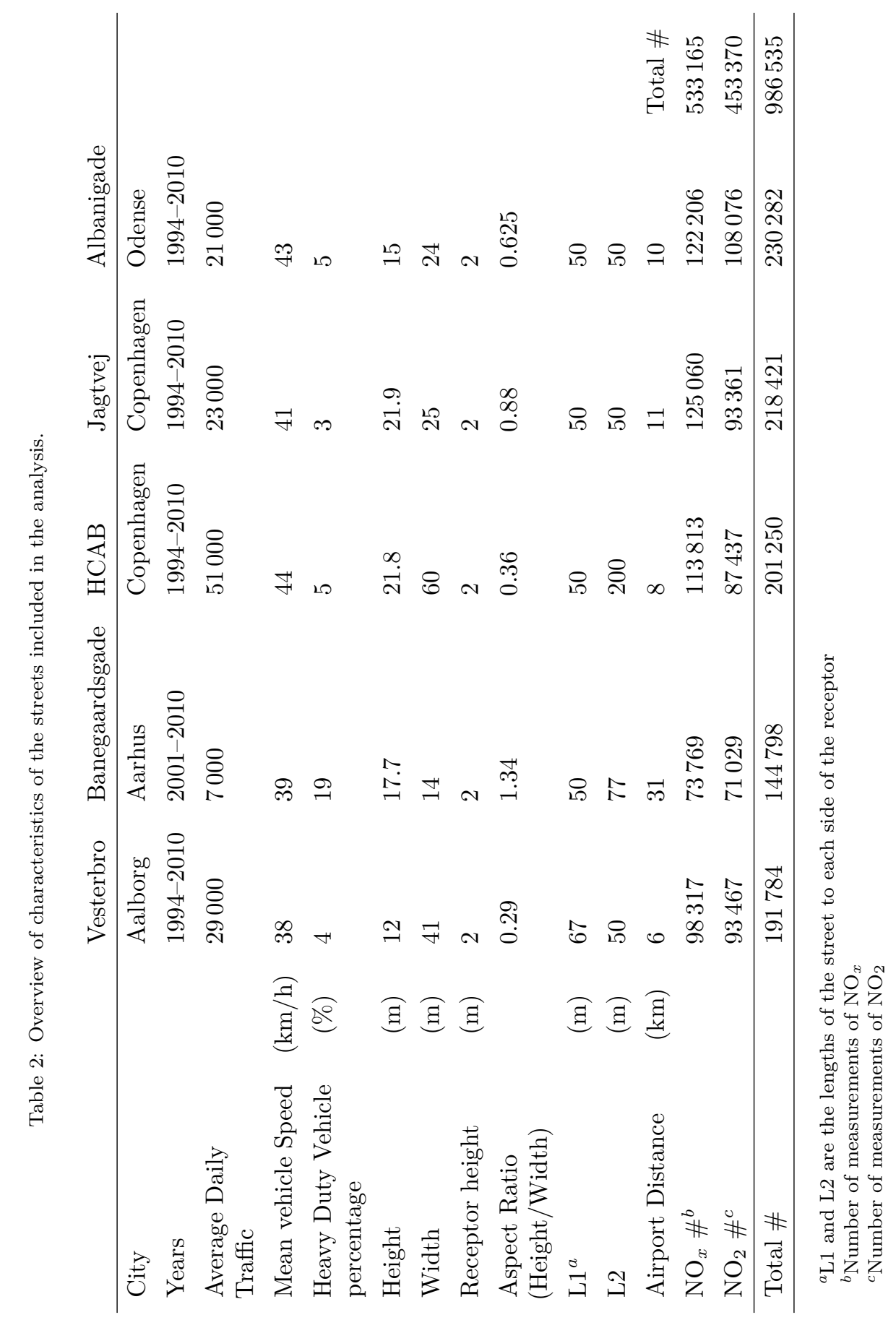


is estimated to $2 \%$ and $\mathrm{NO}_{2}$ to $5 \%$ on a $95 \%$ confidence interval ${ }^{2}$ (Based on results in Skov et al. (1997); the laboratory holds an EN 17025 accreditation). The term measurement uncertainty is used as defined in ISO 20988:2007 Air quality - Guidelines for estimating measurement uncertainty where the measurement uncertainty is calculated from measurement uncertainty budgets and represents the total measurement uncertainty on the measurand. Thus in the present study the measurement uncertainty (as mentioned to be $2 \%$ for $\mathrm{NO}_{x}$ and $5 \%$ for $\mathrm{NO}_{2}$ ) is considered to be negligible compared to model uncertainty.

Details of the traffic input to the analysis can be found in the supplementary material.

\subsection{Data preparation}

In order for the applied methodology to give meaningful results, proper data preparation has to be performed. For the present study outlier detection, data splitting ${ }^{3}$ and pseudo-replicate ${ }^{4}$ removal were analysed as potential data preparation strategies. The description of these data preparation techniques is collected and summarized in the supplementary material. The exact application of the data preparation techniques to the present study is likewise described in the supplementary material.

\subsection{Identifiability analysis}

In order to be able to asses the identifiability of the model parameters, a reasonable estimate of the parameters has to be defined in the prior analysis. In the present case, the original model parameters were chosen since they have shown good performance in earlier validation studies (Ketzel et al., 2011, 2012). The identifiability measures are summed up in Table 3 (Brun et al., 2001). Based on literature (Brun et al., 2002, 2001; Ruano et al., 2007; Sin et al., 2010; Sin and Vanrolleghem, 2007a) a collinearity index threshold of 10 was chosen.

\footnotetext{
${ }^{2}$ The Uncertainty is referring to the general EN 17025 standard and more specifically to EN 14211:2012 Ambient air. Standard method for the measurement of the concentration of nitrogen dioxide and nitrogen monoxide by chemiluminescence

${ }^{3}$ using two data splitting methodologies known as the DUPLEX and seasonal data split.

${ }^{4}$ Identical or almost identical data points.
} 
Table 3: Overview of the applied identifiability analysis. $y_{l}$ stands for the $l$ th model variable, $\theta_{k}$ stands for the $k$ th model parameter, and $S C_{l}$ is a scaling factor for the variable $y_{l} . S C_{l}$ is used to normalize the sensitivities of the different model outputs, and in the present study the mean value of the model output is used. $K$ stands for the index of the parameter subset, which is a combinatorial function of the parameter vector, $\theta$. Table modified from Sin et al. (2010) based on Brun et al. (2002, 2001).

\begin{tabular}{|c|c|c|c|}
\hline Steps: & Description: & & \\
\hline Absolute sensitivity & $S_{a}=\left\{s_{\mathrm{a}, \mathrm{lk}}\right\}$ & where & $s_{\mathrm{a}, \mathrm{lk}}=\left(\frac{\partial y_{l}}{\partial \theta_{k}}\right)_{\theta_{i \neq l}}$ \\
\hline Non dimensional sensitivity & $S_{n d}=\left\{s_{\mathrm{nd}, \mathrm{lk}}\right\}$ & where & $s_{\mathrm{nd}, \mathrm{lk}}=\frac{\partial y_{l}}{\partial \theta_{k}} \cdot \frac{\theta_{k}}{\mathrm{SC}_{l}}$ \\
\hline Sensitivity measure, $\delta^{\mathrm{msqr}}$ & $\delta^{\mathrm{msqr}}=\sqrt{\frac{1}{N} \sum_{l=1}^{N}\left(s_{\mathrm{nd}, \mathrm{lk}}\right)^{2}}$ & & \\
\hline Normalized sensitivity & $S_{\text {norm }}=\left\{s_{\text {norm }, \mathrm{lk}}\right\}$ & where & $s_{\mathrm{norm}, \mathrm{lk}}=\frac{s_{\mathrm{nd}, \mathrm{lk}}}{\left\|s_{\mathrm{nd}, \mathrm{lk}}\right\|}$ \\
\hline Collinearity index, $\gamma_{K}$ & $\begin{aligned} \gamma_{K} & =\frac{1}{\sqrt{\min \lambda_{K}}} \\
\lambda_{K} & =\operatorname{eigen~}\left(s_{\text {norm }, \mathrm{K}}^{T} s_{\text {norm }, \mathrm{K}}\right)\end{aligned}$ & where & \\
\hline
\end{tabular}

The sensitivities of the model to changes in the parameters were calculated using the finite difference approximation. The size of the perturbation factor for the individual parameters was determined using the method of De Pauw (2005).

\subsection{Parameter Estimation}

For the parameter estimation, the non-linear regression approach as described by Seber and Wild (1989) was used. The fundamental assumption is that the measurements can be described as independent random variables with a normally distributed associated probability. These random variables are assumed to have mean values equal to the exact model results and known standard deviations. If this is the case, maximizing the probability of modelling the measurements corresponds to minimizing the difference between model and measurements $\left(\chi^{2}\right)$ :

$$
\chi^{2}=\sum_{i=1}^{n} W_{i} \frac{\left(y_{\text {meas } \left., \mathrm{i}-y_{M, i}\left(\mathbf{P}_{M}\right)\right)^{2}}\right.}{\sigma_{\text {meas }, \mathrm{i}}^{2}}
$$

Where $n$ is the number of measurements, $y_{\text {meas,i }}$ is the measurements, $y_{M, i}\left(\mathbf{P}_{M}\right)$ is the model results as a function of the parameter vector $\mathbf{P}_{M}, M$ is the number of parameters in the model, $\sigma_{\text {meas, }}^{2}$ is the variance of the corresponding measurement, and $W_{i}$ is the weight assigned to the individual observation. 
The above theoretical framework was originally designed for laboratory experiments, and the above assumptions can thus in general not be expected to be valid when transferred to models in environmental science. In laboratory experiments the experiments can be repeated. As described in Section 2.2, the same measurement of respectively $\mathrm{NO}_{x}$ or $\mathrm{NO}_{2}$ was not repeated in the present study. The variance of the individual measurement was therefore substituted by the mean value of the measurement of the individual species. This is a common approach applied in e.g. Silver et al. (2013). In this way, it was avoided that the pollutants appearing in high concentrations dominated over the pollutants appearing in low concentrations.

As shown in the supplementary material, the amount of available measurements vary between streets and for different pollutants. The weight $\left(W_{i}\right)$ was thus used to assign equal weight to the two species in the analysis. The discussion of different weights applied to the different streets was assessed to be outside the scope of this work and thus no street dependent weighing was applied.

To minimize Eq. (1) an iterative minimization procedure in the form of a generalized pattern search algorithm (MathWorks Inc., 2013) was used. This was applied through the "patternsearch" function of the Matlab Global Optimization Toolbox. Bounds were imposed on the individual parameters based on physical principles in order to ensure physically realistic solutions.

\subsection{Exploration of potential bias problems}

Snee (1977), quoting Draper and Smith (1966), recommended analysing the stability of the fitted parameters by splitting the data into each year and fitting the data to each split. In this way, an indication of whether the parameters are constants or functions of other parameters can be obtained. Moreover, this also gives an indication of the parameter spread and the temporal variation in the parameters. For the present case, the years 2004-2010 were chosen since all five streets have measurements in these years. Furthermore, there are approximately the same number of $\mathrm{NO}_{x}$ and $\mathrm{NO}_{2}$ measurements in the data set for these years. The data have furthermore been split according to street, wind speed, and atmospheric stability. 
Both data splitting algorithms, the parameter estimation and the identifiability analysis have been implemented in Matlab by the authors. To implement the DUPLEX algorithm (Snee, 1977) for a large dataset as used in the present study, various computational techniques had to be applied. These are described in the supplementary material. The identifiability analysis was based on the source code from the study by Sin et al. (2010).

\section{Results and discussion}

Following the methodology illustrated in Fig. 1, the results of the local sensitivity analysis (step 5 and 6) are presented in Sections 3.1 and 3.2. The results of the identifiability analysis (step 7 and 8) are found in Sections 3.3 and 3.4, and the results of the parameter estimation (step 9) are described in Section 3.5. Lastly, the bias and sources of error (step 10) are explored in Section 3.6.

\subsection{Determination of perturbation factor to use in the local sensitivity analysis}

The local sensitivity analysis, and thereby the calculation of $\delta^{\mathrm{msqr}}$ and the collinearity index $\gamma_{K}$, is based on the finite difference approximation. In order for the subsequent analyses to be as valid as possible, the calculated derivatives have to be as close to linear as possible. As shown by De Pauw (2005); De Pauw and Vanrolleghem (2003), the optimal perturbation interval can be parameter and output dependent. Using the same methodology the perturbation factor used in the finite difference approximation was analysed by calculating the sum of squared errors (SSE) between the positive and negative perturbation as a function of perturbation interval from $10^{-1}$ to $10^{-7}$.

A set of perturbation factors for the individual parameters were chosen. This was done based on a visual inspection of the plot of error functions, as a function of perturbation interval, for respectively $\mathrm{NO}_{x}$ and $\mathrm{NO}_{2}$. The perturbation factors were chosen to be smaller than $10^{-1}$ in order for the sensitivity analysis to remain local. The same perturbation factor was used for both $\mathrm{NO}_{x}$ and $\mathrm{NO}_{2}$ since the error functions turned out to be relatively similar. If the minimum of the error function was at two different values for the two output parameters, the mean 
Table 4: Optimal perturbation factors for the individual model parameters calculated on the DUPLEX data split. Similar results were obtained for the seasonal data split.

\begin{tabular}{ll} 
Parameter: & Perturbation factor: \\
\hline$\alpha$ & 0.1 \\
$c$ & 0.01 \\
$L_{t}$ & 0.1 \\
$d$ & 0.1 \\
$f_{\text {roof }}$ & 0.05 \\
$h_{0}$ & 0.1 \\
$z_{0}$ & 0.1 \\
$g$ & 0.01 \\
$i$ & 0.1 \\
$S_{p}$ & 0.05 \\
$S_{t}$ & 0.1 \\
$b$ & 0.01 \\
$k$ & 0.1 \\
$\gamma$ & 0.1 \\
\hline
\end{tabular}

value was chosen following the approach of De Pauw (2005). An overview of the perturbation factors for the DUPLEX data split for the model parameters is shown in Table 4.

The results presented in Table 4 are in general larger than the results reported by De Pauw (2005). The reason is that OSPM, compared to the differential equation models analysed by De Pauw (2005), is not very sensitive to changes in the parameters.

The above analysis was as well performed for the data splits of streets, wind speeds, and atmospheric stabilities with similar results as shown in Table 4.

\subsection{Local sensitivity analysis}

For a model to be identifiable, as defined by Brun et al. (2001), the model has to be sensitive to minute changes in parameter values.

A box plot of the relative local sensitivities of the model with respect to concentrations of $\mathrm{NO}_{x}$ is shown in Fig. 2 in descending order of sensitivity $\left(\delta^{\mathrm{msqr}}\right)$ for the DUPLEX data split. 


\section{Relative sensitivity for $\mathrm{NO}_{\mathrm{x}}$}

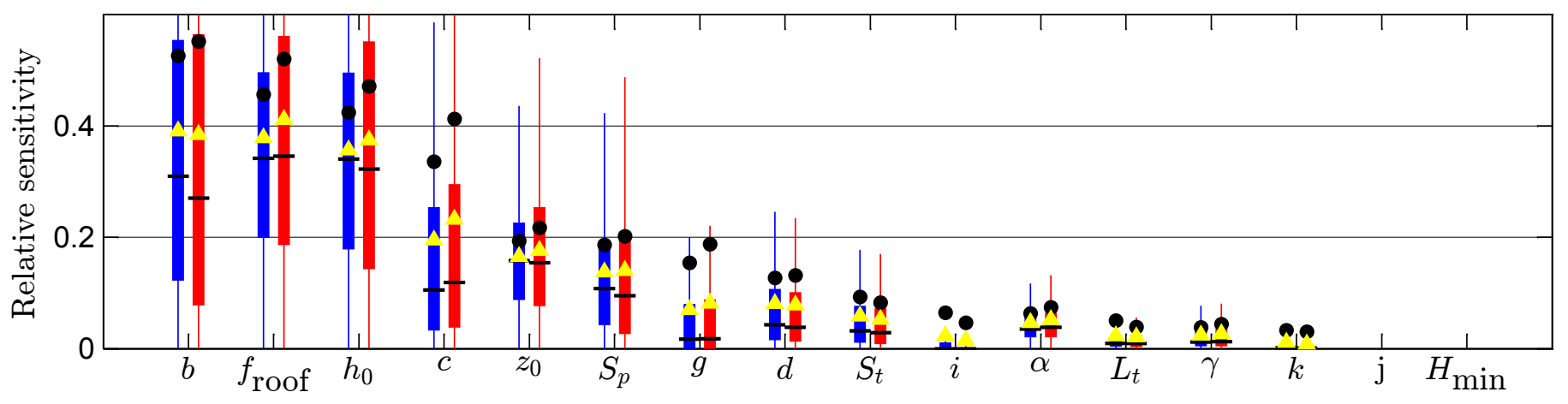

Figure 2: Box plots of the absolute values of the local (relative) non-dimensional sensitivities of the $\mathrm{NO}_{x}$ concentrations to a change in model parameters. The results of the DUPLEX data split are plotted in blue and of the seasonal data split in red. The boxes are the 25th and 75 th percentile, the whiskers correspond to $99.3 \%$ of a normal distribution, the black lines are the median, the mean value of the distribution is represented by yellow triangles, and $\delta^{\text {msqr }}$ is plotted as black dots

The model is most sensitive to changes in the parameter $b$, the scale factor for traffic produced turbulence. $b$ is an important model parameter since it figures in the calculation of street level turbulence, and as such, it influences the calculation of every data point.

$f_{\text {roof }}$, the factor reducing the wind speed from the mast to the roof level, also has a large influence on the model output. $f_{\text {roof }}$ has been the subject of a previous examination (Silver et al., 2013) and influences the street level wind speed and thus all the data points. For $\mathrm{NO}_{2} f_{\text {roof }}$ is the most sensitive model parameter (results for $\mathrm{NO}_{2}$ are found in the supplementary material).

As can be seen from Fig. 2, the parameter $h_{0}$, the initial dispersion height of the plume in the wake of a car, is also a very sensitive model parameter. This means that the concentrations are assumed to be homogeneously mixed below the level of $h_{0}$. The receptor height on all five streets is set to $2 \mathrm{~m}$ corresponding to the value of $h_{0}$. This feature is increasing the model sensitivity to $h_{0}$. Moreover, $h_{0}$ influences the street level wind speed such that the larger $h_{0}$ the larger the street level wind speed compared to the roof level wind speed. I can thus be seen that the most sensitive parameters in a semi-parametric air pollution model 
are the ones affecting the street level wind speed and turbulence.

It can likewise be seen that the model is very sensitive to changes in $c . c$ determines the length of the recirculation zone and thus the integration length of the direct contribution. This is especially important for wide streets and near parallel wind directions. In these situations the length of the recirculation zone is not reduced due to the presence of a downwind building. However, as can be seen from Fig. 2 the parameter $c$ has a very large spread, with a low number of points increasing the value of $\delta^{\mathrm{msqr}}$ substantially. The importance of this parameter might therefore be overestimated using the $\delta^{\mathrm{msqr}}$ criterion. This feature is repeating for many of the model parameters, and indicates that there is a large variability in the model parameter sensitivity.

In general, the sensitivity of the parameters is declining approximately linearly. This is in opposition to the results found by Sin et al. (2010) where the model output was very sensitive to a few model parameters and almost insensitive to the rest of the parameters. This difference could be explained by the difference in model, but is more likely the result of a difference in the data points used for calculating the sensitivity. The present analysis is built on a large data set with good coverage of the different situations the model is designed to handle. This means that all model parameters influence at least some data points (with the exception of $j$ and $H_{\min }$, the two parameters controlling the general building height, where the model has zero sensitivity for all perturbation factors). This is a general feature of large data sets, where the coverage of different situations is better. Therefore, most model parameters have a sensitivity proportional to their influence in the data set.

Figure 2 could indicate that the local sensitivity is marginally larger in the seasonal data split compared to the DUPLEX data split. This is caused by the larger spread in the seasonal data split indicating the more heterogeneous composition of the dataset. The general trend between sensitive and less sensitive model parameters is however reproduced in both data splitting approaches. 
The local sensitivity of the parameters with respect to street and wind speed was as well explored, and the results are reported in the supplementary material.

\subsection{Correlation analysis}

A heat map of the correlation matrix for the DUPLEX and seasonal data split is presented in Fig. 3. The parameters controlling the general building height $\left(j\right.$ and $\left.H_{\text {min }}\right)$ are excluded from the figure due to zero sensitivity. The model parameters with correlation higher than 0.5 have been highlighted in red colours to illuminate potential identifiability issues.

A general look at Fig. 3 shows that only a few parameters have potential identifiability problems. This is again in opposition to the results found by Sin et al. (2010). The difference is most likely once again due to the large data set used for the present analysis. This means that parameters that are related in the model do not compensate each other numerically due to differences in input parameters.

$h_{0}$ and $z_{0}$ appear in the same formula in the model and that the two parameters show correlation is therefore not surprising. The same is true for $b, S_{p}$, and $S_{t}$. The correlations among these sets of parameters is so high that it could indicate identifiability issues.

The correlation between $f_{\text {roof }}$ and $g$ is because both parameters have similar wind direction dependencies. The two parameters follow approximately the same pattern for Vesterbro, Banegaardsgade, and Albanigade whereas they are diverging more for HCAB and Jagtvej (results shown in the supplementary material). This is thus not assessed to constitute an identifiability problem.

The correlation between $f_{\text {roof }}$ and $i$ comes from the dependence of the averaging interval for wind speeds lower than $i$. Indirectly, these two parameters are thus linked in the same equation for calculating the averaging interval.

There is also a noticeable correlation between $\gamma$ and $\alpha$; however, the sensitivity of $\gamma$ is so low that the correlation between the two parameters is not considered the largest identifiability issue for these parameters. 


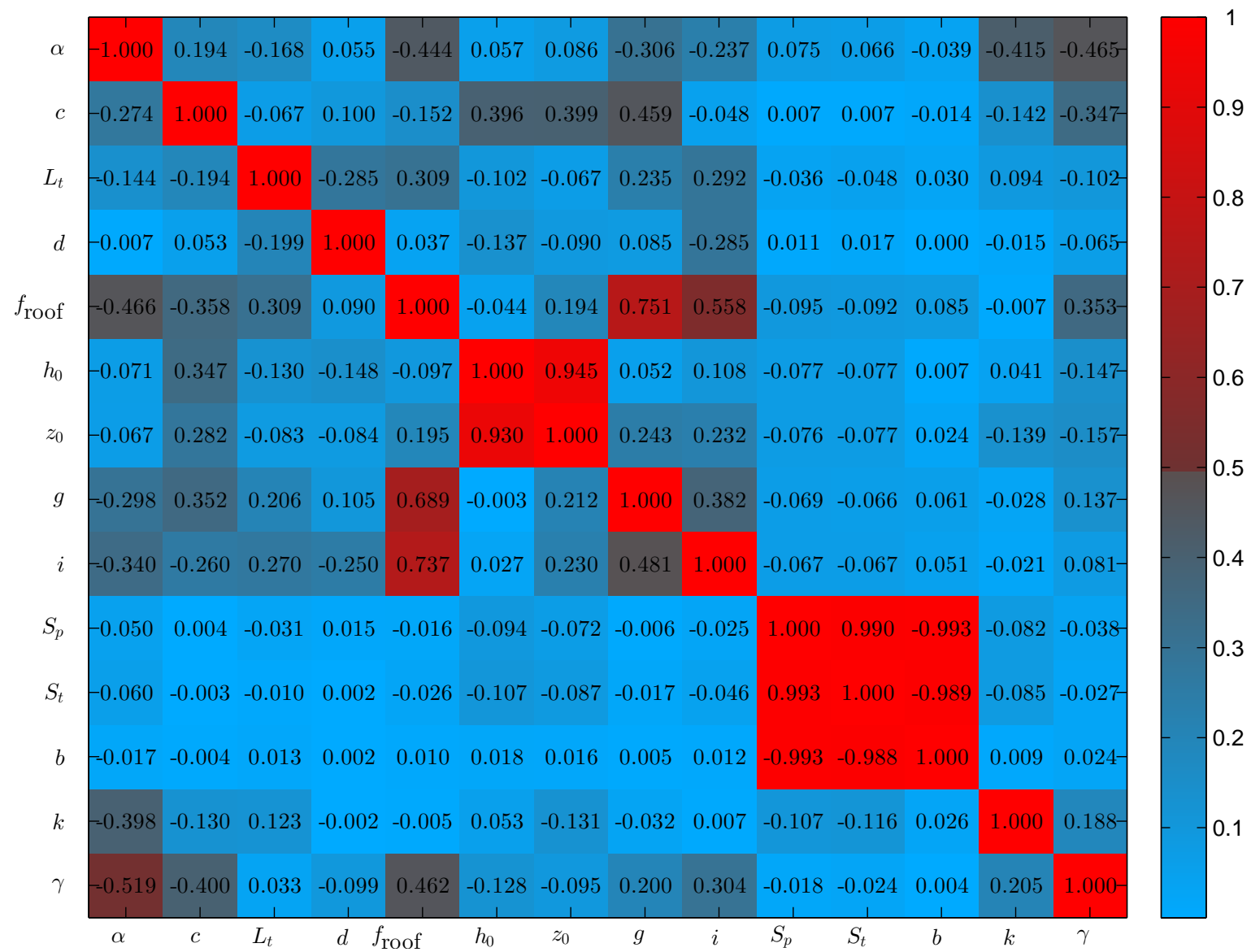

Figure 3: Heatmap of the correlation matrix for the DUPLEX data split (lower triangular matrix) and the seasonal data split (upper triangular matrix). Correlations smaller than \pm 0.5 are plotted on the blue part of the colour scale and correlations larger than \pm 0.5 are plotted on the red part of the colour scale. Only parameters with non-zero sensitivity are included in this analysis.

\subsection{Collinearity analysis}

The results of the calculation of the collinearity index $\gamma_{K}$ for all parameter subsets are shown in Table 5. As can be seen, the more parameters included the more collinear the combination becomes. This is obvious since including more parameters means a better chance of a change in one parameter being compensated by a change in the other parameters. Especially the lower boundary of the $\gamma_{K}$ range is increasing whereas the upper boundary is almost stable from three parameters and upwards. As can be seen, the largest number of parameters with $\gamma_{K}<10$ is 12 ; however, several different combinations of 12 parameters are identifiable. 
By comparing Table 5 to Table 1, it can be seen that the least collinear parameters are the ones representing very different aspects of the model. E.g. the parameter $c$ describes the recirculation zone, and thereby the direct contribution, and the parameter $i$ represent the limit for increased wind direction averaging. This is also seen by that the related parameters for traffic produced turbulence $S_{p}, S_{t}$, and $b$ have high collinearities.

Among the combinations of 12 parameters with a collinearity index lower than 10 , the parameters with collinearity problems were $h_{0}$ and $z_{0}$; and $S_{t}, S_{p}$, and $b$, as also seen from Fig. 3. The combinations of $f_{\text {roof }}$ and respectively $g$ and $i$ and the combination of $\alpha$ and $\gamma$, that indicated identifiability problems in Fig. 3, are therefore correlated without being collinear, a phenomenon also observed by Brun et al. (2001) in a different branch of science. $z_{0}$ is a standard value used in many different atmospheric models. It was therefore assessed that fixing this parameter would not mean a great loss of information in the analysis. Moreover, this parameter also has a lower sensitivity compared to $h_{0}$.

For the traffic produced turbulence the parameter $S_{t}$ was set fixed. This was chosen because the model has the lowest sensitivity to this parameter across the dataset among the three parameters related to traffic produced turbulence. In order to generate comparative results, the same parameters were estimated for the seasonal data split. The same combination of parameters also satisfy the collinearity criterion for the seasonal data split.

\subsection{Parameter Estimation}

The results of the parameter estimation for the DUPLEX and the seasonal data split are shown in Table 6 in the order of decreasing $\delta^{\mathrm{msqr}}$ for the DUPLEX data split for $\mathrm{NO}_{x}$. From the table it can be seen that the two data splitting approaches have resulted in quite different sets of parameter values.

The two data split have approximately $43 \%$ shared data points, but analysing the histograms of the unique part of the two sets show no significant differences. There is, however, a trend towards that the seasonal data split has a more repre- 
Table 5: Collinearity results for different subsets of the DUPLEX data split

\begin{tabular}{rrrrl}
\hline Size $^{a}$ & $\begin{array}{c}\text { Combi- } \\
\text { nations }^{b}\end{array}$ & $\gamma_{K}$ range $^{c}$ & $\gamma_{K}<10(\%)^{d}$ & Parameters subset for $\gamma_{\text {min }}$ \\
\hline 2 & 91 & $0.71-9.29$ & 100.0 & $c, L_{t}$ \\
3 & 364 & $0.78-42.28$ & 97.8 & $c, L_{t}, i$ \\
4 & 1001 & $0.84-42.30$ & 93.3 & $c, L_{t}, i, k$ \\
5 & 2002 & $1.00-42.30$ & 86.8 & $d, g, i, k, \gamma$ \\
6 & 3003 & $1.07-42.33$ & 78.4 & $\alpha, L_{t}, g, i, k, \gamma$ \\
7 & 3432 & $1.13-42.43$ & 68.4 & $\alpha, L_{t}, d, g, i, k, \gamma$ \\
8 & 3003 & $1.53-42.48$ & 56.6 & $\alpha, L_{t}, d, g, i, S_{t}, k, \gamma$ \\
9 & 2002 & $2.08-42.50$ & 43.7 & $\alpha, L_{t}, d, g, i, S_{p}, S_{t}, k, \gamma$ \\
10 & 1001 & $2.66-42.52$ & 30.2 & $\alpha, c, L_{t}, d, g, i, S_{p}, S_{t}, k, \gamma$ \\
11 & 364 & $4.92-42.52$ & 17.3 & $\alpha, c, L_{t}, d, z_{0}, g, i, S_{p}, S_{t}, k, \gamma$ \\
12 & 91 & $8.41-42.53$ & 6.6 & $\alpha, c, L_{t}, d, f_{\text {roof }}, z_{0}, g, i, S_{p}, S_{t}, k, \gamma$ \\
13 & 14 & $20.47-42.54$ & 0.0 & $\alpha, c, L_{t}, d, f_{\text {roof }}, h_{0}, z_{0}, g, i, S_{t}, b, k, \gamma$ \\
14 & 1 & $42.54-42.54$ & 0.0 & $\alpha, c, L_{t}, d, f_{\text {roof }}, h_{0}, z_{0}, g, i, S_{p}, S_{t}, b, k, \gamma$ \\
\hline
\end{tabular}

${ }^{a}$ Size of the parameter combination set

${ }^{b}$ Total number of combinations of a given size

${ }^{c}$ Highest and lowest value for $\gamma_{K}$

${ }^{d}$ Percentage of combinations with a $\gamma_{K}$ value less than 10

Table 6: Parameter estimates for both the DUPLEX and the seasonal data split plus confidence intervals for the parameters estimated on the basis of the DUPLEX data splitting procedure.

\begin{tabular}{|c|c|c|c|c|c|c|c|}
\hline$\theta$ & $\begin{array}{c}\text { Original } \\
\text { value }\end{array}$ & & Limits & $\begin{array}{l}\text { Estimate } \\
\text { Seasonal }\end{array}$ & $\begin{array}{l}\text { Estimate } \\
\text { DUPLEX }\end{array}$ & \% Difference & $\begin{array}{l}95 \% \text { CL } \\
\% \text { of mean } \theta\end{array}$ \\
\hline$b$ & 0.3 & & {$\left[10^{-5}: 0.999\right]$} & 0.212 & 0.288 & 30.5 & \pm 0.6 \\
\hline$f_{\text {roof }}$ & 0.4 & & {$\left[10^{-5}: 0.999\right]$} & 0.427 & 0.422 & 1.2 & \pm 1.2 \\
\hline$h_{0}$ & 2.0 & $\mathrm{~m}$ & {$[0.6: 10]$} & 2.177 & 1.422 & 42.0 & \pm 0.9 \\
\hline$c$ & 2.0 & & {$[0.25: 10.00]$} & 6.607 & 5.685 & 15.0 & \pm 1.5 \\
\hline$S_{P}$ & 2.0 & $\mathrm{~m}^{2}$ & {$\left[10^{-5}: 10\right]$} & 1.198 & 0.360 & 107.5 & \pm 2.5 \\
\hline$g$ & 2.0 & $\frac{\mathrm{m}}{\mathrm{s}}$ & {$\left[10^{-5}: 10\right]$} & 0.116 & 0.085 & 31.5 & \pm 18.5 \\
\hline$d$ & 0.5 & & {$\left[10^{-5}: 2 \pi\right]$} & 1.873 & 1.105 & 51.6 & \pm 0.3 \\
\hline$i$ & 1.0 & $\frac{\mathrm{m}}{\mathrm{s}}$ & {$\left[10^{-5}: 10\right]$} & 0.455 & 0.713 & 44.3 & \pm 1.4 \\
\hline$\alpha$ & 0.1 & & {$[0.05: 2.00]$} & 0.277 & 0.292 & 5.5 & \pm 1.0 \\
\hline$L_{t}$ & 0.5 & & {$\left[10^{-5}: 0.999\right]$} & 0.008 & $1.335 \cdot 10^{-5}$ & 199.3 & $\pm 1.3 \cdot 10^{5}$ \\
\hline$\gamma$ & 0.2 & & {$\left[10^{-5}: 0.999\right]$} & 0.789 & 0.017 & 191.5 & \pm 52.1 \\
\hline$k$ & 0.4 & & [0.04:0.999] & 0.999 & 0.999 & 0.0 & \pm 8.7 \\
\hline
\end{tabular}


sentative coverage of the data points. The DUPLEX data split has conversely a better coverage of the input parameter space.

Likewise, an analysis of the correlation between the input data show no significant difference in correlation structure for the two data set. However, the input data of the seasonal data split tends to be more correlated with each other.

It can be seen from Table 6 that the percentage difference (with a few exceptions) is much larger than the $95 \%$ confidence interval. This is an indication that the $95 \%$ confidence interval, calculated via linear error propagation, underestimates the uncertainty on the parameters. The cause of the underestimation is the linear approximation applied to a non linear model in the calculation of the $95 \%$ confidence interval (Joshi et al., 2006). Whether the percentage difference is representative for the uncertainty on the model parameters, in the form of e.g. the standard deviation, could be examined through a bootstrapping approach (Efron, 1979; Wu et al., 2012); however, this was deemed infeasible due to the long run times of the parameter estimation algorithm.

From Table 6 a trend can be seen towards higher percentage difference on the parameters with low sensitivity. This is natural since these parameters have to change significantly for the least squares to be reduced. Comparing the results of Table 6 with Fig. 2 shows that, the parameters being estimated to the limits of the estimation interval are the ones with very low sensitivity.

The wind speed parametrization in OSPM, connecting the parameters $f_{\text {roof }}$, $h_{0}$, and $\gamma$, means that one should be careful in the interpretation of the parameter uncertainties. $f_{\text {roof }}$ has an almost constant minimum close to the original model value. This leaves the other two parameters to determine the street level wind speed. Increasing $\gamma$ will lead to a lower street level wind speed (at least for perpendicular wind directions); whereas, increasing $h_{0}$ will lead to increasing street level wind speeds. Comparing the result of the two data splitting approaches shows exactly this phenomenon. An analysis of the relative difference in wind speed between the two sets of parameters shows that it is approximately $12 \%$. This is valid for the input data from both the DUPLEX and the seasonal data 
split. This shows that the difference in wind speed is smaller than the difference in parameters as indicated in Table 6 .

The parameter $c$ has increased substantially in both data splitting approaches. The fitted parameters of 6-6.5 building heights should not be taken as the physical length of the recirculation zone. Rather, this should be seen as a parameter yielding the best fit to data. Referring to Table 2, the only two streets with sufficient lengths to accommodate a recirculation zone of six building heights are Vesterbro and HCAB. Increasing $c$ will lead to increased concentrations, especially for near parallel wind directions. Increasing the value of $\alpha$ will cancel this effect to a certain extent. This will at the same time reduce the concentration of $\mathrm{NO}_{2}$ due to the decreased residence time in the canyon.

The results of the parameter estimation on the weekly diurnal concentration profile can be found in the supplementary material.

\subsection{Exploration of potential bias problems}

In the following sections an exploration of the parameters estimated in Section 3.5 is presented. The general validity of the parameters, in the form of their respective identifiability, is presented in Section 3.6.1. The stability of the parameter estimates with respect to street geometry and traffic is analysed in Section 3.6.2. The performance of the parameters with respect to the weekly diurnal variation and atmospheric stability is analysed in the supplementary material, and the stability of the parameters with respect to wind speed is analysed in Section 3.6.3.

\subsubsection{Identifiability Analysis}

The relative local sensitivity of the original and estimated parameters are presented in Fig. 4. The parameter estimation procedure has significantly altered the relative sensitivity of the parameters. Some parameters such as $b, f_{\text {roof }}$, and $h_{0}$ are, however, still very sensitive, and parameters such as $L_{t}, k$, and $i$ are still insensitive.

The collinearity index $\gamma_{K}$ for the DUPLEX data split is 10.12 and 12.05 for the seasonal data split. The two sets of parameters can thus be seen not 
Relative sensitivity for $\mathrm{NO}_{\mathrm{x}}$

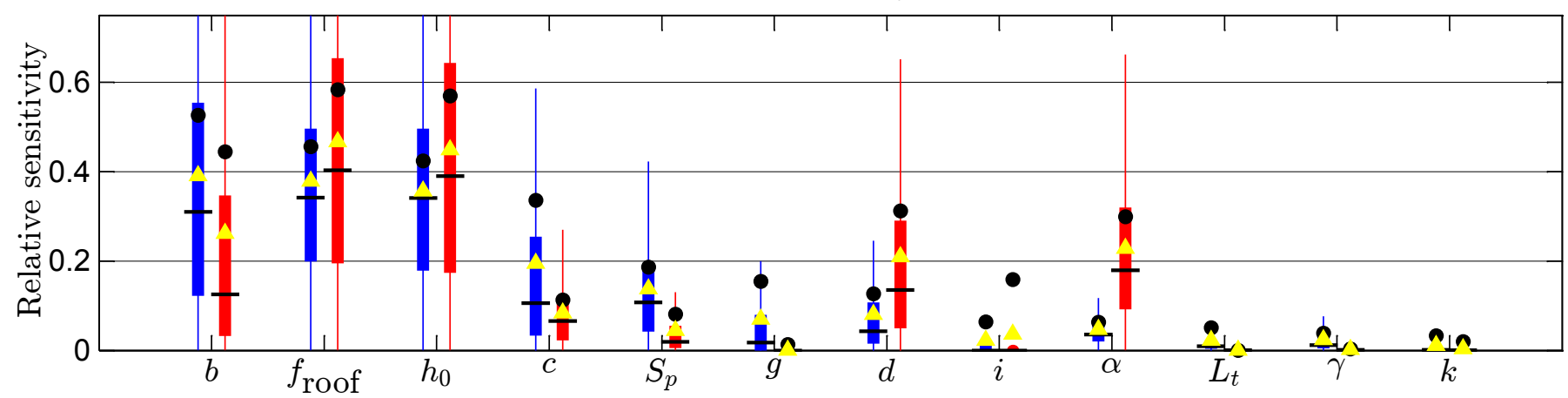

(a)

Relative sensitivity for $\mathrm{NO}_{\mathrm{x}}$

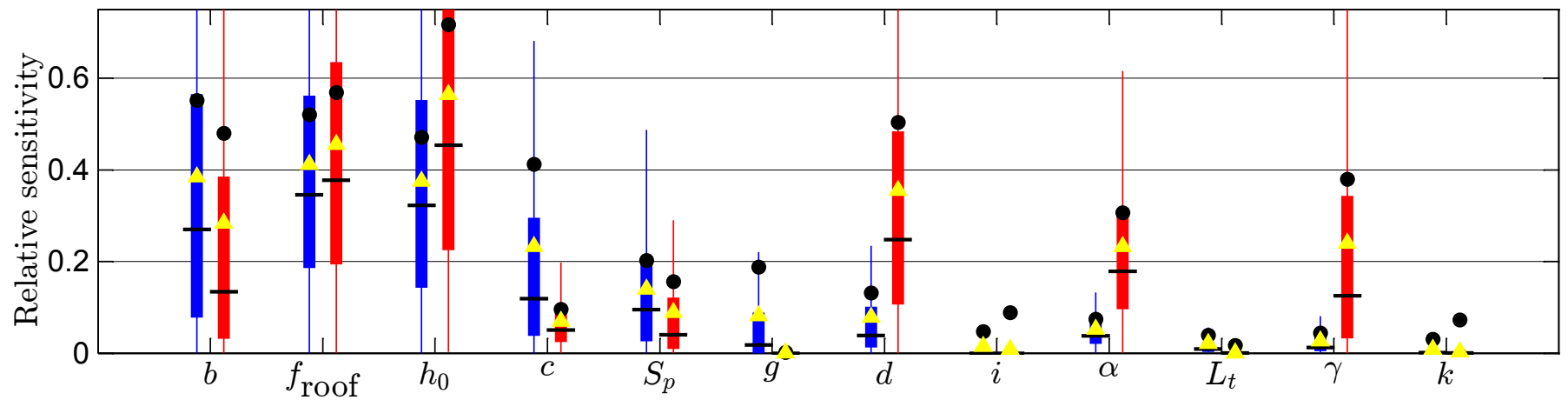

(b)

Figure 4: Comparison of the relative sensitivity for $\mathrm{NO}_{x}$ before (blue) and after (red) parameter estimation. Figure $4 \mathrm{a}$ are for the DUPLEX data split and corresponding parameter values, and Fig. 4b are for the seasonal data split. The blue colour represent the original model parameters and the red colour represent the estimated parameters. The box plots are designed similarly to Fig. 2.

to be collinear measured on their respective data set. The collinearity indices for the two data split are slightly above the limit set in Section 2.4. This was, however, deemed acceptable since the limit on collinearity has a certain element of subjectivity in it.

\subsubsection{Influence of street geometry}

The correlation coefficient $\left(R^{2}\right)$, the fractional bias (FB), and the normalised mean square error (NMSE) for the two data splits and the individual streets without data splitting for the $\mathrm{NO}_{x}$ concentrations are shown in Fig. 5 and for $\mathrm{NO}_{2}$ in Fig. 6. 

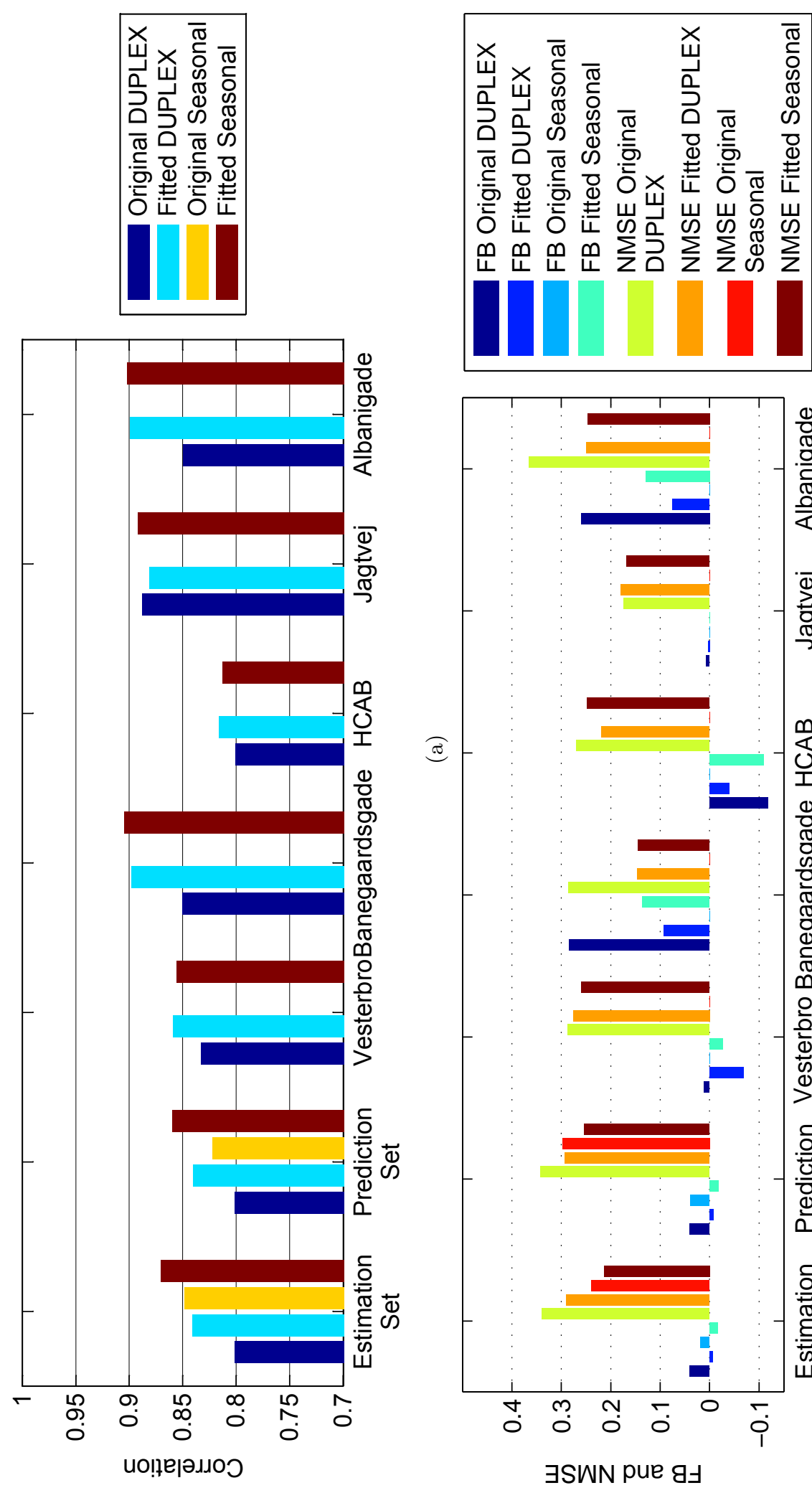

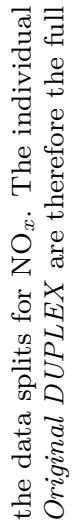

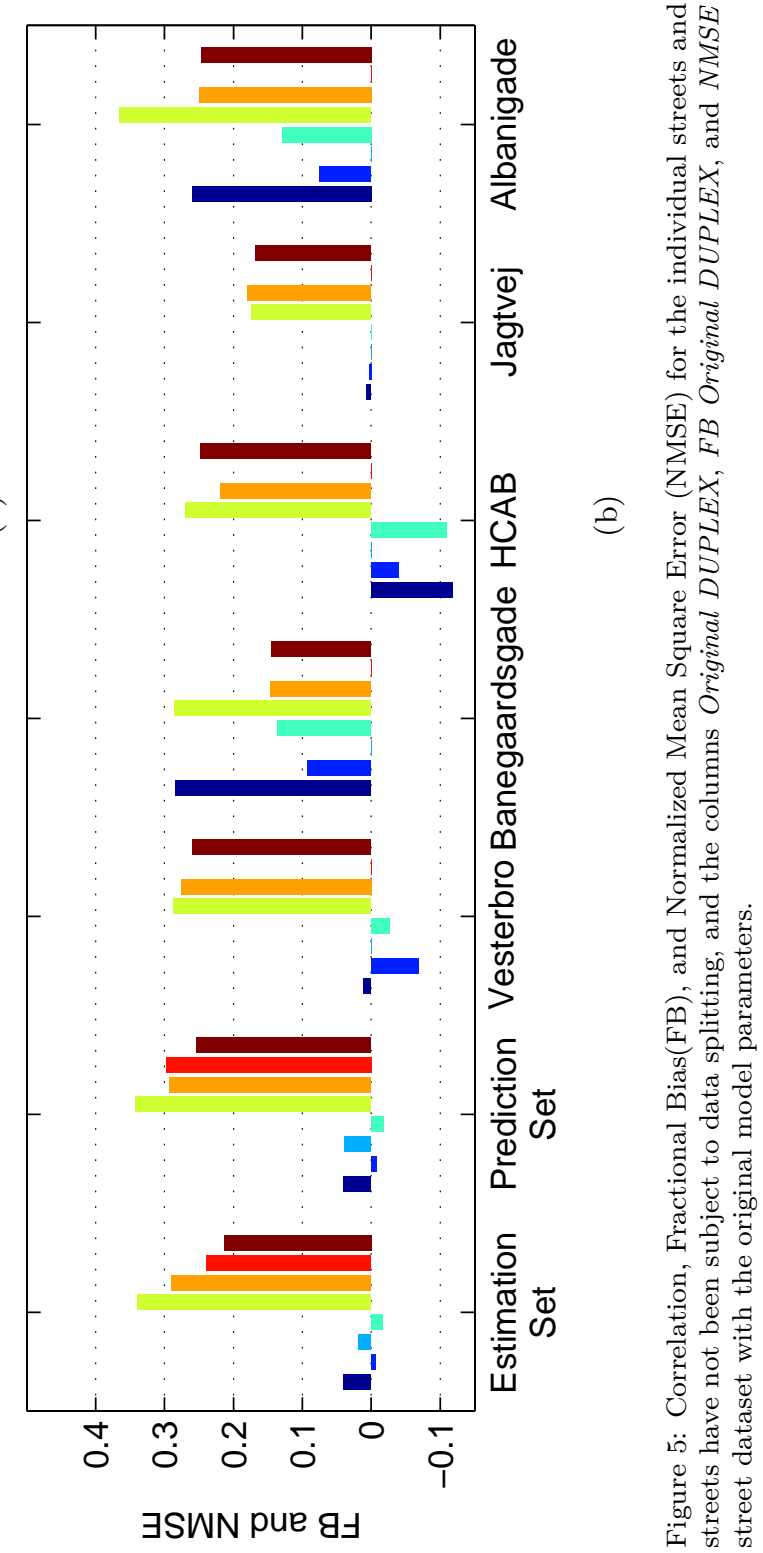



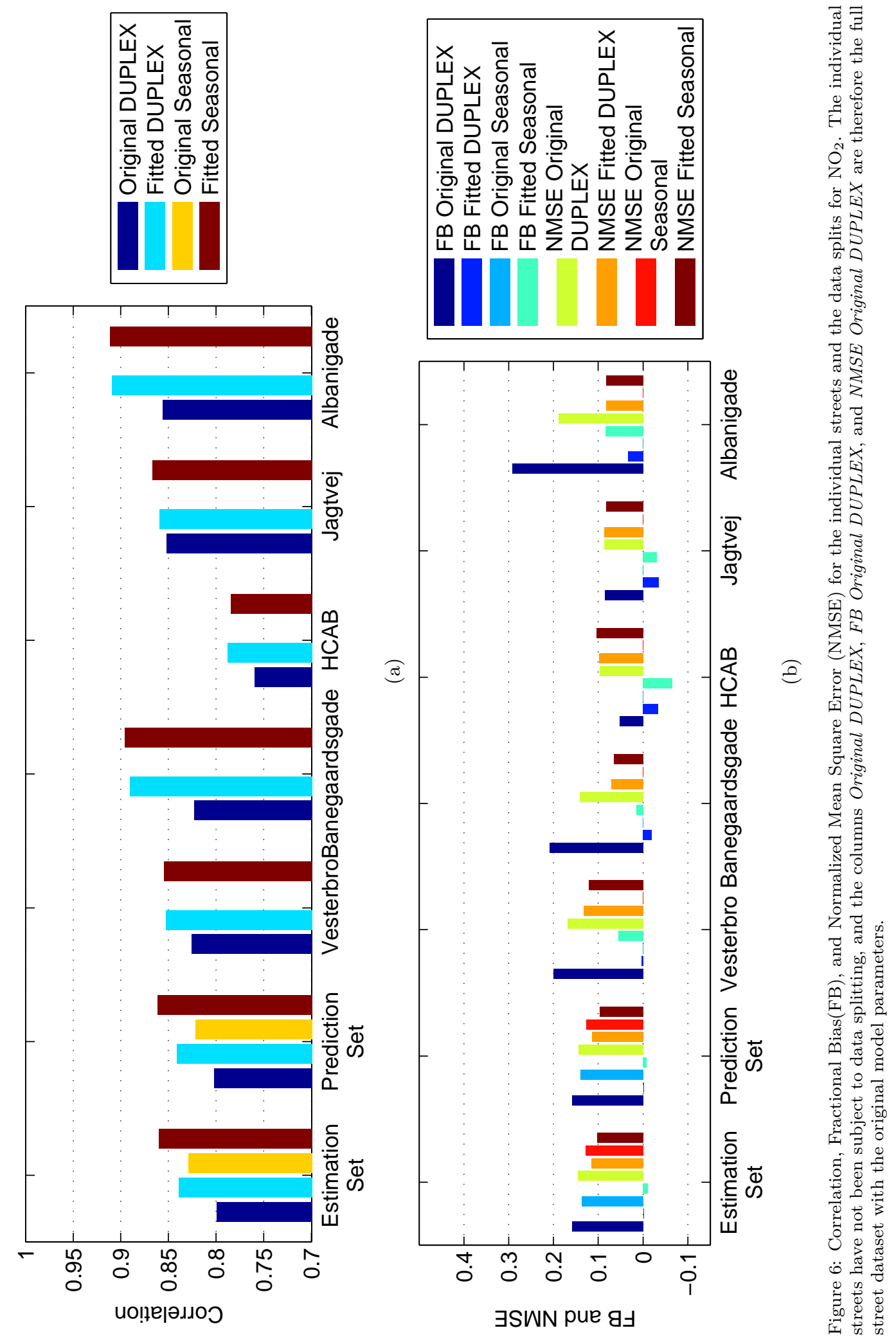
As can be seen, for both estimation and prediction sets, the estimated parameters perform noticeably better than the original model parameters. This is seen for all three statistical quantities. This is especially the case for $\mathrm{NO}_{2}$ where the large fractional bias in the original model parameters has disappeared. For the DUPLEX data split, the statistical quantities are almost identical from the estimation to the prediction set. Contrary to this, the seasonal data split has noticeably lower performance for the prediction set compared to the estimation set on all three statistical parameters. For the individual streets it can be seen that there are some significant differences in the model performance across the streets. The highest and lowest correlation coefficient for the original model parameters are respectively found for Jagtvej and HCAB. The low correlation coefficient for HCAB is caused by the physics governing irregular street canyons not being properly accounted for in the model. This also means that there is not much model improvement using parameter estimation for this street, as also seen on Figs. 5 and 6. For Jagtvej the correlation coefficient for $\mathrm{NO}_{x}$ has declined slightly as a result of the parameter estimation. However, the overall trend is still towards model improvement considering that the statistics for the vast majority of the other streets have increased significantly. This indicates that the model could in the past have been calibrated against $\mathrm{NO}_{x}$ data from Jagtvej. The results of this being optimal performance for this street on behalf of less optimal performance for the other streets and the other species. The parameter estimation scheme has thus served to distribute the errors homogeneously among the individual street canyons and the individual species.

The parameter estimation procedure was repeated on the individual streets (results found in the supplementary material). The results indicate that the uncertainty on the model parameters is larger than indicated by the percentage difference in Table 6 . The parameters obtained in Table 6 can thus be seen as average parameters converging when averaged over many streets with different properties. Moreover, this indicates that the model parameters depend in complicated ways on the street geometry and the traffic conditions on the individual street. More accurate results could thus be obtained by making the parameters dependent on street geometry and traffic conditions. 
Overall, it can be seen from Figs. 5 and 6 that the DUPLEX and seasonal estimated parameter sets perform approximately equally well on the various streets. The phenomenon, that two or more parameter sets give approximately equal model performance, has been coined equifinality by Beven (2006). The phenomenon arises in the interplay between model and measurements. Here, the combination of model input uncertainty, model structural uncertainty, and model parameter uncertainty is converted to uncertainty in the fitted parameter values. None of the aforementioned types of uncertainty have previously been assessed for OSPM and the present input data before. It is therefore difficult to judge how much of the variance is caused by which form of uncertainty.

The equifinality of the model and the measurements appears to be a type of identifiability problem, also sometimes known as non-uniqueness (Beven, 2002). This type of identifiability problem has not been accounted for by the identifiability analysis applied in the present study. The identifiability analysis of Brun et al. (2001) is based on local sensitivity, and as such, is not designed to analyse this type of global identifiability problems. One could thus argue that a smaller subset of parameters should have been estimated. However, to the best of the authors knowledge, there exists no method to determine a globally identifiable parameter subset. Moreover, this would make the estimated parameters dependent on the (poorly defined?) fixed parameters.

\subsubsection{Influence of wind speed}

The correlation coefficient $\left(R^{2}\right)$, the fractional bias (FB), and the normalized mean square error (NMSE) of the model with estimated (based on the DUPLEX and seasonal data split) and original model parameters for $\mathrm{NO}_{x}$ are shown in Fig. 7. As can be seen, the original model parameters have a systematic bias as a function of wind speed. The FB is almost zero for wind speeds of $1 \frac{\mathrm{m}}{\mathrm{s}}-3 \frac{\mathrm{m}}{\mathrm{s}}$, but higher for calm winds and higher wind speeds. The NMSE of the original model parameters are also increasing sharply as the wind speed increases to more than $3 \frac{\mathrm{m}}{\mathrm{s}}$. The FB of the estimated model parameters is also increasing with higher wind speeds albeit at a much slower rate. The same trend is also found to a 

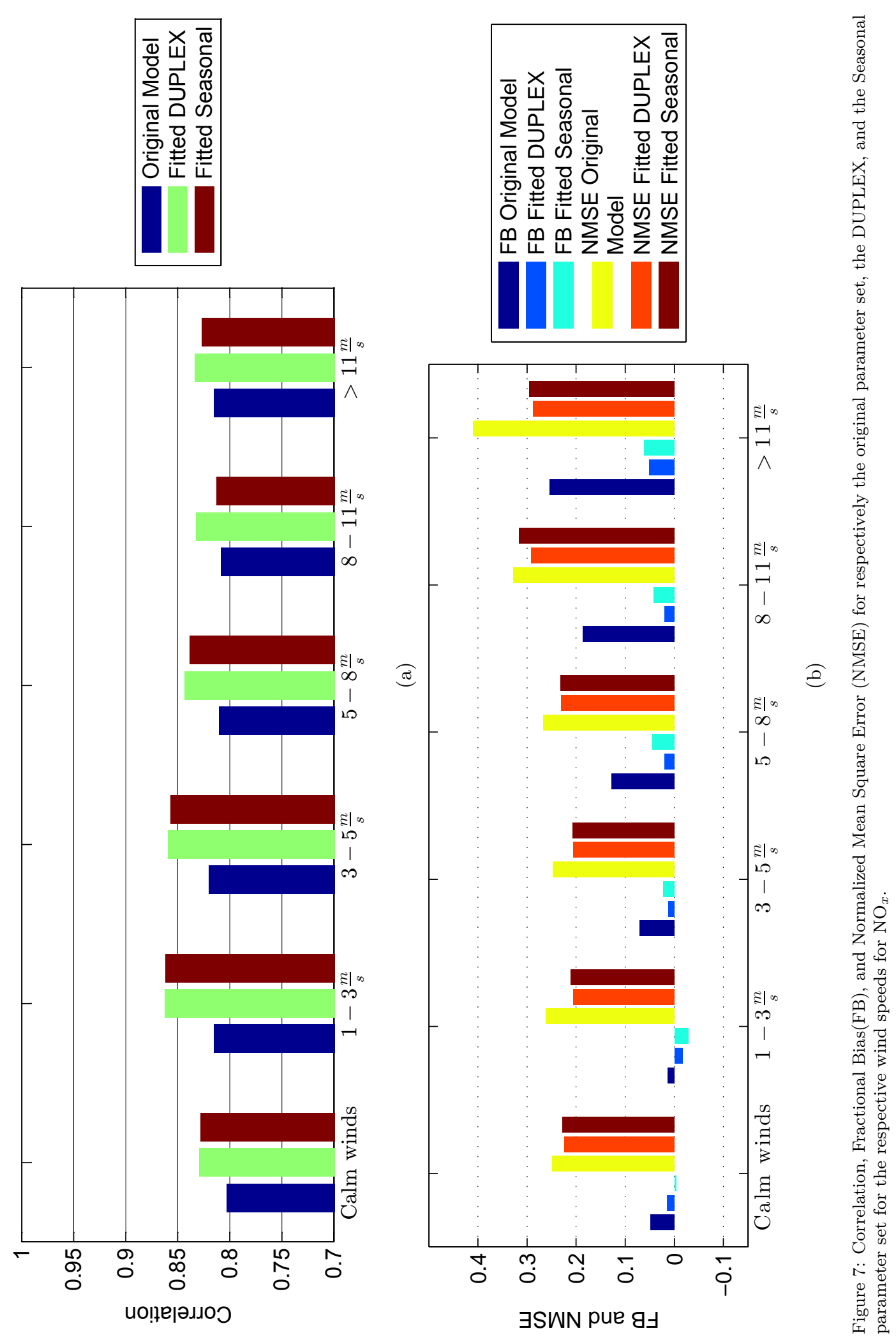
lesser extent in the correlation coefficient. Again, this shows that the parameter estimation procedure equilibrates the errors among the different situations the model is exposed to.

The results of the parameter estimation applied to the respective wind speed classes can be found in the supplementary material.

\section{Conclusions}

In this study, it is shown that it is possible to apply the methodology of Brun et al. (2001) to atmospheric models and obtain informative results that can be used for reduction of model outcome uncertainty. It was shown in Section 3.6.2 that the parameter estimation procedure successfully equilibrated the bias among the individual streets and among the individual species. This should be compared to the more heterogeneous performance of the original model parameters. Thus it was shown that this methodology could serve to improve this type of model.

When applied to the two data splitting approaches, the methodology revealed that the estimated parameters were much more uncertain than indicated by their $95 \%$ confidence intervals. This shows that the frequentist approach to uncertainty analysis, as applied here, tends to underestimate the uncertainty of the parameters. Other methods, such as bootstrapping or Bayesian approaches to uncertainty analysis, should be used to validate the results of the frequentist approach. The methodology of Brun et al. (2001) do not include application to different realisations of the data set, but does neither preclude this element. It is therefore recommended that a bootstrapping approach is used for the parameter estimation part of the methodology.

The large uncertainty of the parameters were confirmed by fitting the parameters to the individual streets and the individual years. As discussed in Section 3.6, the large uncertainties could be interpreted as an identifiability problem. It was however shown in the local sensitivity analysis and the extensive validation performed in this study that there could be various ways to reduce the large uncertainty. This could be done through reductions in model structural 
uncertainty and model input uncertainty besides model parameter uncertainty. In this way, the applicability and advantage of the methodology for this type of model is shown.

\section{Acknowledgements}

The HPC resources and services used in this work were provided by the IT Research Computing group in Texas A\&M University at Qatar. IT Research Computing is funded by the Qatar Foundation for Education, Science and Community Development (http://www.qf .org.qa).

This publication was made possible by a NPRP award [NPRP 7-674-2-252] from the Qatar National Research Fund (a member of The Qatar Foundation). The statements made herein are solely the responsibility of the authors.

The authors would like to express their gratitude to Claus Nordstrøm for input on how the manual outlier removal was performed. Furthermore, the authors would like to thank Steen Solvang Jensen for input on how the traffic profiles were generated. Lastly the authors would like to thank Jeremy D. Silver for valuable comments and feedback to the article.

\section{References}

Anh, D. T., M. P. Bonnet, G. Vachaud, C. V. Minh, N. Prieur, L. V. Duc, and L. L. Anh (2006). Biochemical modeling of the Nhue River (Hanoi, Vietnam): Practical identifiability analysis and parameters estimation. Ecological Modelling 193(3-4), $182-204$

Bei, N., G. Li, and L. T. Molina (2012). Uncertainties in SOA simulations due to meteorological uncertainties in Mexico City during MILAGRO-2006 field campaign. Atmospheric Chemistry and Physics 12(23), 11295-11308.

Berkowicz, R. (2000). OSPM - A Parameterised Street Pollution Model. Environmental Monitoring and Assessment 65(1-2), 323-331.

Berkowicz, R., O. Hertel, S. Larsen, N. N. Sørensen, and M. Nielsen (1997). Modelling traffic pollution in streets. Technical report, Ministry of Environment and Energy, National Environmental Research Institute. 
Beven, K. (2002). Towards a coherent philosophy for modelling the environment. Proceedings of the Royal Society of London A 458, 2465-2484.

Beven, K. (2006). A manifesto for the equifinality thesis. Journal of Hydrology 320(1-2), 18-36.

Brun, R., M. Kühni, H. Siegrist, W. Gujer, and P. Reichert (2002). Practical identifiability of AMS2d parameters - systematic selection and tuning of parameter subsets. Water Research 36, 4113-4127.

Brun, R., P. Reichert, and H. R. Künsch (2001). Practical identifiability analysis of large environmental simulation models. Water Resources Research 37(4), 1015-1030.

De Pauw, D. (2005). Optimal Experimental Design for Calibration of Bioprocess Models: a Validated Software Toolbox. Ph. D. thesis, Faculteit BioIngenieurwetenschappen. Universiteit Gent. http://dx.doi.org/1854/4975.

De Pauw, D., K. Steppe, and B. D. Baets (2008). Identifiability analysis and improvement of a tree water flow and storage model. Mathematical Biosciences 211(2), $314-332$.

De Pauw, D. J. W. and P. A. Vanrolleghem (2003). Practical aspects of sensitivity analysis for dynamic models. In Proceedings: IMACS 4th MATHMOD Conference. February 5-7, 2003, Vienna, Austria, pp. 328-336.

Draper, N. R. and H. Smith (1966). Applied Regression Analysis. John Wiley \& Sons, New York.

Efron, B. (1979). Bootstrap Methods: Another Look at the Jackknife. The Annals of Statistics 7(1), 1-26.

Ellermann, T., J. K. Nøjgaard, C. Nordstrøm, J. Brandt, J. Christensen, M. Ketzel, S. Jansen, A. Massling, and S. S. Jensen (2013). The Danish Air Quality Monitoring Programme. Annual Summary for 2012. Technical Report Scientific Report from DCE. no. 67, Arhus University, DCE - Danish Centre for Environment and Energy. 
EMEP/EEA (2009). EMEP/EEA air pollutant emission inventory guidebook. Technical Report No 9/2009, European Environment Agency. Www.eea.europa.eu/publications/ emep-eea-emission-inventory-guidebook-2009, accessed 23 November 2013 .

Franke, J., A. Hellsten, H. Schlünzen, and B. Carissimo (2007). Best Practice Guideline for the CFD Simulation of Flows in the Urban Environment-Quality Assurance and Improvement of Microscale Meteorological Models. COST Action 732 .

Freni, G., G. Mannina, and G. Viviani (2009). Identifiability analysis for receiving water body quality modelling. Environmental Modelling \& Software 24(1), 54 -62 .

Hanna, S. R. (2007). A review of uncertainty and sensitivity analyses of atmospheric transport and dispersion models. In C. Borrego and E. Renner (Eds.), Air Pollution Modelling and Its Applications, Volume XVIII. Elsevier, Amsterdam, The Netherlands.

Hanna, S. R., R. Paine, D. Heinold, E. Kintigh, and D. Baker (2007). Uncertainties in Air Toxics Calculated by the Dispersion Models AERMOD and ISCST3 in the Houston Ship Channel Area. Journal of Applied Meteorology and Climatology 46(9), 1372-1382.

Hertel, O. and R. Berkowicz (1989a). Modelling $\mathrm{NO}_{2}$ Concentrations in a Street Canyon. Technical Report 131, National Environmental Research Institute. DMU LUFT-A.

Hertel, O. and R. Berkowicz (1989b). Modelling Pollution from Traffic in a Street Canyon. Evaluation of Data and Model Development. Technical Report 129, National Environmental Research Institute. DMU LUFT-A.

Hertel, O. and R. Berkowicz (1989c). Operational Street Pollution model (OSPM). Evaluation of the Model on Data from St. Olavs Street in Oslo. Technical Report 135, National Environmental Research Institute. DMU LUFT-A. 
Joshi, M., A. Seidel-Morgenstern, and A. Kremling (2006). Exploiting the bootstrap method for quantifying parameter confidence intervals in dynamic systems. Metabolic Engineering 8, 447-455.

Ketzel, M., R. Berkowicz, M. Hvidberg, S. S. Jensen, and O. Raaschou-Nielsen (2011). Evaluation of AirGIS: A GIS-based air pollution and human exposure modelling system. International Journal of Environment and Pollution $47(1 / 2 / 3 / 4), 226-238$.

Ketzel, M., S. S. Jensen, J. Brandt, T. Ellermann, H. R. Olesen, R. Berkowicz, and O. Hertel (2012). Evaluation of the Street Pollution Model OSPM for Measurements at 12 Streets Stations Using a Newly Developed and Freely Available Evaluation Tool. Journal of Civil \& Environmental Engineering S:1.

Manomaiphiboon, K. and A. G. Russell (2004). Effects of uncertainties in parameters of a Lagrangian particle model on mean ground-level concentrations under stable conditions. Atmospheric Environment 38(33), 5529-5543.

Marsik, T. and R. Johnson (2010). Model for Estimation of Traffic Pollutant Levels in Northern Communities. Journal of the Air 83 Waste Management Association 60, 1335-1340.

Martinez-Lopez, B., S. Peyron, N. Gontard, and M. Mauricio-Iglesias (2015). Practical identifiability analysis for the characterization of mass transport properties in migration tests. Industrial \&3 Engineering Chemistry Research 54(17), $4725-4736$.

MathWorks Inc. (2013). Global Optimization Toolbox User's Guide. Webpage. www . mathoworks.com/help/gads/patternsearch.html.

Meier, W. K. and P. Reichert (2005). Mountain streams-modeling hydraulics and substance transport. Journal of Environmental Engineering 131(2), 252 261.

Muñoz, E., D. Rivera, F. Vergara, P. Tume, and J. L. Arumí (2014). Identifiability analysis: towards constrained equifinality and reduced uncertainty in a conceptual model. Hydrological Sciences Journal 59(9), 1690-1703. 
Omlin, M., R. Brun, and P. Reichert (2001). Biogeochemical model of lake zürich: sensitivity, identifiability and uncertainty analysis. Ecological Modelling 141(1-3), $105-123$.

Ottosen, T.-B., K. E. Kakosimos, C. Johansson, O. Hertel, J. Brandt, H. Skov, R. Berkowicz, and M. Ketzel (2015). Implementation and Validation of an Inhomogeneous Emission Geometry Scheme in a Semi-Parametrized Street Canyon Model. Geoscientific Model Development 8, 3231-3245.

Palmgren, F., R. Berkowicz, O. Hertel, and E. Vignati (1996). Effects of reduction of $\mathrm{NO}_{x}$ on the $\mathrm{NO}_{2}$ levels in urban streets. The Science of the Total Environment 189/190, 409-415.

Ruano, M., J. Ribes, D. J. W. De Pauw, and G. Sin (2007). Parameter subset selection for the dynamic calibration of activated sludge models (ASMs): experience versus systems analysis. Water Science and Technology 56(8), $107-115$.

Seber, G. A. F. and C. J. Wild (1989). Nonlinear Regression. John Wiley \& Sons.

Silver, J. D., M. Ketzel, and J. Brandt (2013). Dynamic parameter estimation for a street canyon air quality model. Environmental Modelling $\mathcal{E} 3$ Software 47 , $235-252$.

Sin, G., A. S. Meyer, and K. V. Gernaey (2010). Assessing reliability of cellulose hydrolysis models to support biofuel process design - Identifiability and uncertainty analysis. Computers and Chemical Engineering 34, 1385-1392.

Sin, G. and P. A. Vanrolleghem (2007a). Extensions to modeling aerobic carbon degradation using combined respirometric-titrimetric measurements in view of activated sludge model calibration. Water Research 41, 3345-3358.

Sin, G. and P. A. Vanrolleghem (2007b). Extensions to modeling aerobic carbon degradation using combined respirometric-titrimetric measurements in view of activated sludge model calibration. Water Research 41(15), $3345-3358$. 
Skov, H., A. H. Egeløv, K. Granby, and T. Nielsen (1997). Relationships between ozone and other photochemical products at Ll. Valby, Denmark. Atmospheric Environment 31(5), 685-691.

Snee, R. D. (1977). Validation of Regression Models: Methods and Examples. Technometrics 19(4), 415-428.

Vardoulakis, S., B. E. A. Fisher, N. Gonzalez-Flesca, and K. Periclous (2002). Model sensitivity and uncertainty analysis using roadside air quality measurements. Atmospheric Environment 36, 2121-2134.

Walker, W. E., P. Harremoës, J. Rotmans, J. P. van Der Sluijs, M. B. A. van Asselt, P. Janssen, and M. P. K. von Krauss (2003). Defining Uncertainty a Conceptual Basis for Uncertainty Management in Model-Based Decision Support. Integrated Assessment 4(1), 5-17.

Wu, W., R. May, G. C. Dandy, and H. R. Maier (2012). A method for comparing data splitting approaches for developing hydrological ANN models. In 2012 International Congress on Environmental Modelling and Software. Managing Resources of a Limited Planet: Pathways and Visions under Uncertainty, Sixth Biennial Meeting, pp. 1620-1627. International Environmental Modelling and Software Society (iEMSs). http://www. iemss .org/society/index.php/ iemss-2012-proceedings. 